\title{
Diet-induced obesity mediates a proinflammatory response in pancreatic $\beta$ cell via toll-like receptor 4
}

\author{
JUAN LI ${ }^{1}$, SHUFEN CHEN ${ }^{1}$, JUAN QIANG ${ }^{1}$ XIN WANG ${ }^{1}$, LEI CHEN ${ }^{2}$, DAJIN ZOU ${ }^{3}$ \\ ${ }^{1}$ Department of Clinical Nutrition, Changhai Hospital, Second Military Medical University, Shanghai, PR China \\ ${ }^{2}$ International Cooperation Laboratory on Signal Transduction, Eastern Hepatobiliary Surgery Institute, Second Military Medical University, \\ Shanghai, PR China \\ ${ }^{3}$ Department of Endocrinology, Changhai Hospital, Second Military Medical University, Shanghai, PR China
}

\begin{abstract}
Toll-like receptor 4 has an important role in inflammation and immunity. Whether TLR4 signaling contributes to the link between insulin resistance and islet $\beta$ cell dysfunction is an unanswered question. Here, we show that in the face of the same high-fat continuous stimulation for 24 weeks, in TLR4-/- HF mice, the weight, fraction of the liver, epididymal fat pad fraction, as well as blood glucose and insulin levels were lower than in the WT HF group. In TLR4-/- HF mice, the $\mathrm{O}_{2}$ consumption, $\mathrm{CO}_{2}$ production and activities were higher than in the WT HF group. Glucose tolerance test, insulin tolerance test and insulin release test suggest that the impaired insulin secretion was significantly improved in TLR4-/HF mice, compared with the WT HF group. In TLR4-/- HF mice, islet $\beta$ cell ultrastructure was not damaged in the face of the same high-fat continuous stimulation, compared to that in the WT HF group. By detecting glucose-stimulated insulin secretion in the primary islet, insulin secretion of TLR4-1-HF mice was better than that of the WT HF group, and in the TLR4-/- HF group, at the mRNA level, islet interleukin $6(I L-6)$, tumor necrosis factor $\alpha(T N F-\alpha)$, and monocyte chemotactic protein $1(M C P-1)$ were significantly lower than in the WT HF group. There was the islet macrophage infiltration in the WT HF group, but no significant macrophage infiltration in the TLR4-/- HF group. These data suggest that the damaged islet functions of the high fat diet-induced obesity mice may be linked to the TLR4 expression level, and the recruitment of macrophages into the islets.
\end{abstract}

Key words: TLR4, insulin resistance, obesity, islet function, cytokine.

(Centr Eur J Immunol 2014; 39 (3): 306-315)

\section{Introduction}

Chronic inflammation is a key feature of insulin resistance and obesity. In conditions of nutrient excess such as obesity and diabetes, elevated free fatty acid (FFA) levels are implicated in the pathogenesis of both inflammation and insulin resistance in a variety of tissues, including endothelial cells [1-4]. At the cellular level, nutrient excess is linked to insulin resistance via activation of IKK and, subsequently, nuclear factor $\kappa \mathrm{B}(\mathrm{NF}-\kappa \mathrm{B})$, a key transcriptional mediator of inflammation [5-7].

The bacterial endotoxin, lipopolysaccharide (LPS), is a potent activator of IKK $\beta$ and NF- $\mathrm{BB}$ in most cell types. The majority of the biological activity of LPS is contained within a moiety ("lipid A") that is acylated with saturated fatty acids, and removal of these fatty acids results in a complete loss of endotoxic activity [8, 9]. Recently, TLR4 was shown to be required not only for LPS-induced inflammatory responses, but for responses to nonbacterial ligands such as lauric acid (C 12:0), a saturated fatty acid $[10,11]$. These in vitro studies suggest that activation of TLR4 by certain FFA species can trigger cellular inflammatory responses. Whether TLR4 signaling contributes to the link among nutrient excess, inflammation, and metabolic dysfunction in vivo is an important unanswered question.

So, to investigate whether TLR4 signaling contributes to the link between insulin resistance and islet $\beta$ cell dysfunction in vivo, here, we had examined change of the weight, fraction of the liver, epididymal fat pad fraction, blood glucose, insulin levels the $\mathrm{O}_{2}$ consumption, as well as $\mathrm{CO}_{2}$ production and activity in TLR4-/- HF mice, and had also investigated the glucose regulation ability, the islet acute insulin secretion capacity and the insulin sensitivity by glucose tolerance test, insulin tolerance test and insulin release test, and the islet $\beta$ cell ultrastructure by the

Correspondence: Prof. Dajin Zou, Department of Endocrinology, Changhai Hospital, Second Military Medical University, Shanghai, PR China, e-mail: Dajinzou1118@163.com 
electron microscopy in TLR4-/- HF mice, and moreover, we had also investigated the expression of inflammatory cytokines in TLR4-/- HF mice, and these data would lay the foundation for developing therapeutic approaches to improve the insulin sensitivity while preserving innate immunity.

\section{Material and methods}

\section{Mice}

All animal care and experimental procedures were approved by the Animal Care and Usage Committee of Changhai Hospital, Second Military Medical University. Only male mice were included in the current study. TLR4-/- mice on a C57BL/6 background and WT mice were fed high-fat diets (D12331; Research Diets Inc.) for 24 weeks. Mice had free access to water, and average daily food intake was assessed weekly in each cage of mice. Food intake was measured daily and used to calculate total energy intake. Animals were weighed, and abdominal fat pads and liver were removed and weighed. All extracted tissues and blood samples were immediately frozen in liquid nitrogen and stored at $-80^{\circ} \mathrm{C}$.

\section{Isolation of primary pancreatic island}

Briefly, at the end of treatment ( 24 weeks), mice from the four groups were anesthetized by injection with $0.7 \%$ napental into the peritoneal cavity. The tail-end of choledoch proximal to intestine was tied off, and then mice were sacrificed. After the choledoch was cannulated in its tail-end proximal to liver, collagenase solution $(0.5 \mathrm{mg} /$ $\mathrm{ml}$ ) was injected through choledoch. The expanded pancreatic islet was then isolated, put into a culture flask with $6 \mathrm{ml} \mathrm{Krebs}$ solution, and digested at $38^{\circ} \mathrm{C}$ for $10 \mathrm{~min}$. The liquid was sifted by passing through a stainless steel net $(600 \mathrm{~mm})$, and then ice-cold Krebs solution (containing $20 \%$ fetal bovine serum) was added to stop the digestion. The mixture was centrifuged at $250 \mathrm{~g}$ for $1 \mathrm{~min}$ at $4^{\circ} \mathrm{C}$, and the supernatant was discarded. This process was repeated twice. The precipitate was resuspended in $25 \%$ Ficoll solution in a centrifuge tube, and then $23 \%, 20 \%$, and $11 \%$ Ficoll solutions were added carefully on top of each previous layer. After being centrifuged at $500 \mathrm{~g}$ for $15 \mathrm{~min}$ at $4^{\circ} \mathrm{C}$, the interface between $23 \%$ and $20 \%$ Ficoll solutions or between $20 \%$ and $11 \%$ Ficoll solutions was obtained and washed three times with Krebs solution. The obtained pancreatic islet was identified by dithizone.

\section{Body composition analysis}

Body composition (the weight, fraction of the liver, epididymal fat pad fraction) was analyzed using a Bruker's minispec LF50 body composition analyzer according to the manufacturer's instructions (Bruker Optik, Ettlingen, Germany).

\section{Oxygen consumption and $\mathrm{CO}_{2}$ production measurement, and activity counts}

Oxygen consumption and $\mathrm{CO}_{2}$ production were measured in fed animals through an indirect open circuit calorimeter (Oxymax Deluxe System; Columbus Instruments, Columbus, $\mathrm{OH}$ ), and the activities in fed animals were counted at day and night, as described previously [12].

\section{Glucose tolerance test and insulin tolerance tests}

Mice were fasted for $6 \mathrm{~h}$ and were injected intraperitoneally with glucose $(2 \mathrm{~g} / \mathrm{kg})$ for glucose tolerance test (GTT) or for insulin tolerance test (ITT), respectively. Glucose levels were monitored at baseline and at indicated time points after the metabolic challenge via tail-vein blood sampling using a blood glucose meter from Accu-Chek (Roche, Dublin, Ireland). Insulin secretory response was monitored in overnight fasted animals, and blood samples were collected at indicated times after glucose load $(3 \mathrm{~g} / \mathrm{kg}$, Novolin R, ITTS). Insulin levels were measured by enzyme-linked immunosorbent assay (ELISA).

\section{Electron microscopy}

Ultrastructural examination followed standard procedures, as described elsewhere [13]. Briefly, mice from the four groups were first anesthetized by injection with $0.7 \%$ napental into the peritoneal cavity. Pancreatic gland was then isolated, put into ice-cold fixing solution, and fixed at $4^{\circ} \mathrm{C}$ for $24 \mathrm{~h}$. After that, the fixed pancreatic gland was embedded in paraffin, serially sectioned, and detected under a Zeiss EM10 transmission electron microscope (Carl Zeiss, Oberkochen, Germany).

\section{Gene expression analysis by quantitative RT-PCR}

The total RNA was extracted using an RNeasy Mini Kit (QIAGEN), according to the manufacturer's protocol. mRNA expression of inflammatory genes was assessed with quantitative RT-PCR. Briefly, one microgram RNA from each sample was used to generate cDNA using M-MLV reverse transcriptase as per manufacturer's specifications (Promega Corporation, USA). After an initial denaturation step at $95^{\circ} \mathrm{C}$ for 10 min using SYBR Green PCR Master Mix (Applied Biosystems, USA), Real-time PCR was cycled 40 times between $95^{\circ} \mathrm{C} / 15 \mathrm{~s}$ and $60^{\circ} \mathrm{C} / 1$ min. Amplification was performed using 7500 Fast Real-Time PCR Systems (Applied Biosystems, USA) and the products were routinely checked using dissociation curve software. Transcript quantities were compared by the relative $\mathrm{Ct}$ method and the amount of inflammatory genes was normalized to the endogenous control (GAPDH). The value in relation to the control sample was given by $2-\Delta \Delta \mathrm{CT}$. Real-time PCR primer sequences for caspases measure- 

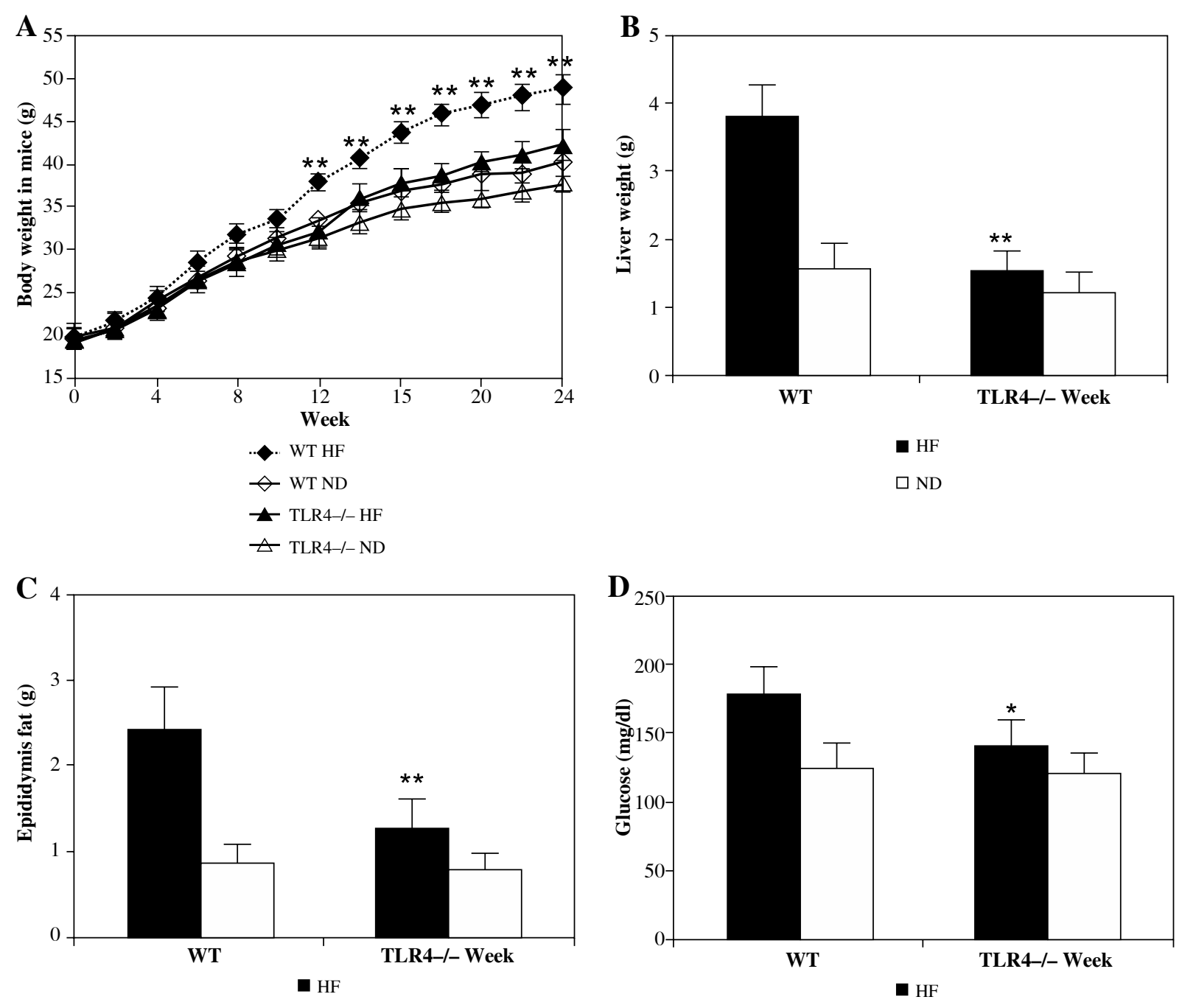

$\square \mathrm{ND}$

$\square \mathrm{ND}$

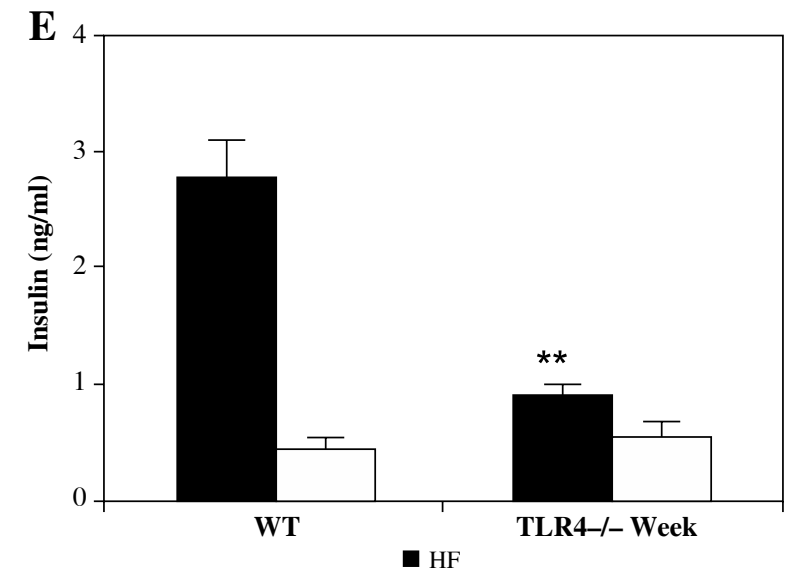

$\square \mathrm{ND}$

Fig. 1. In TLR4-/- HF mice, the weight, fraction of the liver, epididymal fat pad fraction, as well as blood glucose and insulin levels were lower than in the WT HF group. In the four groups, body weight (A) was measured and after 24 weeks, liver fraction $(\mathbf{B})$, epididymal fat pad fraction $(\mathbf{C})$, glucose (D) and fasting insulin $(\mathbf{E})$ were measured. ${ }^{*} p<0.05 ;{ }^{* *} p<0.01$ 
ments were as following: The DNA sequence of primers corresponding to inflammatory genes is as follows: tumor necrosis factor $\alpha(\mathrm{TNF}-\alpha)$, forward, GACCCTCACACTCAGATCATCTTCT, reverse, CCACTTGGTGGTTTGCTACGA; monocyte chemotactic protein 1 (MCP-1), forward, GGCTCAGCCAGATGCAGTTAA, reverse, CCTACTCATTGGGATCATCTTGCT; interleukin 6 (IL-6), forward, TCCAGTTGCCTTCTTGGGACTGAT, reverse, AGCCTCCGACTTGTGAAGTGGTAT.

\section{Immunohistochemistry}

The mice from the four groups were first anesthetized by injection with $0.7 \%$ napental into the peritoneal cavity. Pancreatic gland was then isolated, put into the fixing solution, and pancreatic islets were fixed overnight at room temperature in $10 \%$ zinc-formalin solution and embedded in paraffin. Five micron sections were cut at $50 \mu \mathrm{m}$ intervals and mounted on glass slides, deparaffinized in xylene, and stained for expression of F480 with monoclonal antibody (Serotec, Raleigh, NC) as previously described [14].

\section{Statistical analysis}

Data were reported as means \pm SEM of at least three independent experiments. For statistical analysis, one-way ANOVA was used for comparison of one variance among groups and two-way ANOVA was used for comparison of two independent variances among groups followed by the $t$ test. A $p$ value less than 0.05 was considered to be significant.

\section{Results}

In TLR4-/- HF mice, the weight, fraction of the liver, epididymal fat pad fraction, as well as blood glucose and insulin levels were investigated, compared to those in the WT HF group

As shown in Fig. 1A, after 12 weeks, the body weight of the TLR4-/- HF group was significantly lower than that of the WT HF group, and this trend continued until 24 weeks $(p<0.01)$. In addition, as shown in Fig. 1B, C, D and E, after 24 weeks, the liver fraction (liver / body weight) $(p<0.01)$, epididymal fat pad fraction (epididymal fat/weight $)(p<0.01)$, glucose $(p<0.05)$ and fasting insulin $(p<0.01)$ of the TLR4-/- HF group were significantly lower than those of the WT HF group.

In TLR4-I- HF mice, the $\mathrm{O}_{2}$ consumption, $\mathrm{CO}_{2}$ production and activity were changed, compared to those in the WT HF group

As shown in Fig. 2A, in all groups, there were no significant differences in the food intake. And as shown in Fig. 2B, C, D, E, F and G, in the WT HF group, the oxygen consumption $(p<0.05)$, the amount of emitted carbon dioxide $(p<0.05)$ and activities $(p<0.05)$ were significantly lower than those of the TLR4-/- HF group.

\section{In TLR4-I- HF mice, glucose regulation ability, islet acute insulin secretion capacity, and the sensitivity of insulin were changed, compared to those in the WT HF group}

As shown in Fig. 3A, through glucose tolerance test, 24 weeks after the high fat continued to stimulate, compared with the WT HF group, the blood glucose of TLR4-/group, at $5(p<0.05), 30,60$, and $120(p<0.01)$ minutes was significantly lower; and there were no differences between the WT ND group and the TLR4-/- ND group. These suggest that after the same high-fat diet stimulation, the glucose regulation ability of the TLR4-/- HF group was significantly stronger than that of the WT HF group. As shown in Fig. 3B, the fasting insulin level of WT HF was significantly higher than that of the TLR4-/- HF group, and 2, 5, 15, 30,60 minutes after glucose load, the insulin level was still higher than that of the TLR4-/- ND group; but 2 minutes after the TLR4-/- ND group was injected with sugar, there was the secretion peak with 3-4 times higher than the baseline, while in the WT HF group, the peak was delayed, and the glucose-stimulated acute insulin secretion response decreased $(p<0.05)$. The data suggest that after the sustained high-fat diet stimulation, in WT HF mice, hyperinsulinemia appeared and islet acute insulin secretion was significantly impaired; while after the same high-fat stimuli, the islets acute insulin secretion of TLR4-/- HF mice was protected. To understand the sensitivity to insulin, the insulin tolerance test was done before the injection of insulin, and as shown in Fig. 3C, compared to that of the WT HF group, 30, and 60 minutes after injection of insulin glucose, the blood sugar of TLR4-I- HF decreased significantly, and at 90 minutes, the blood sugar began to rise. These data suggest that the insulin sensitivity of TLR4-/- HF mice was significantly higher than that of the WT HF group.

\section{In TLR4-/- HF mice, islet $\beta$ cell ultrastructure was investigated, compared to that in the WT HF group}

As shown in Fig. 4, using electron microscopy, we found that in WT HF mice, there were islet $\beta$ cell lipid droplets deposition and the increase in the number of insulin secretory vesicles, and the mitochondrial swelling; while in TLR4-/- mice, there were no $\beta$ cell ultrastructure of the above changes, including the deposition of lipid droplets obvious and swelling of mitochondria, similar to $\beta$ cells of the normal diet group, suggesting that in TLR4-/- HF mice, after the same high-fat diet continuous stimulation, the islet $\beta$ cell ultrastructure was not damaged. 
A

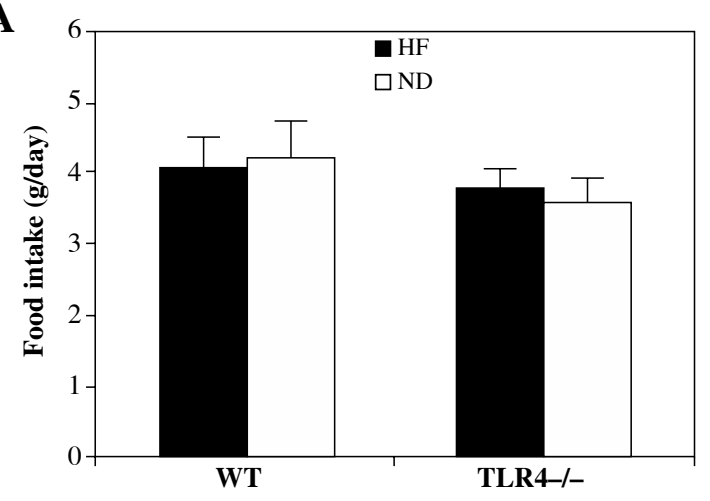

C
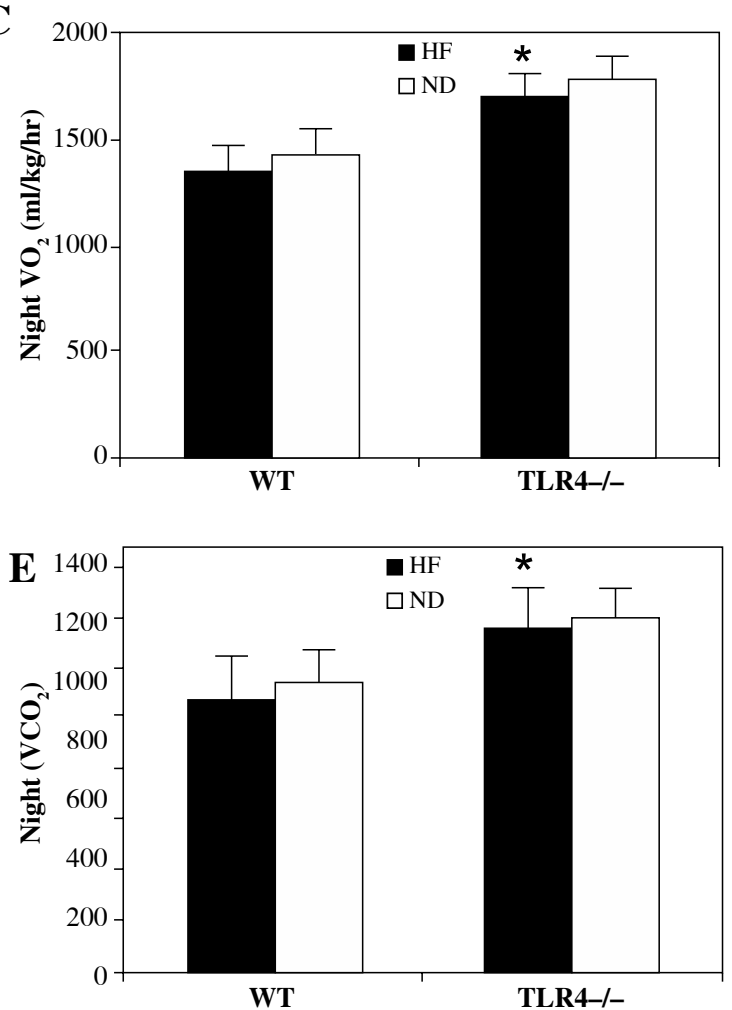

B

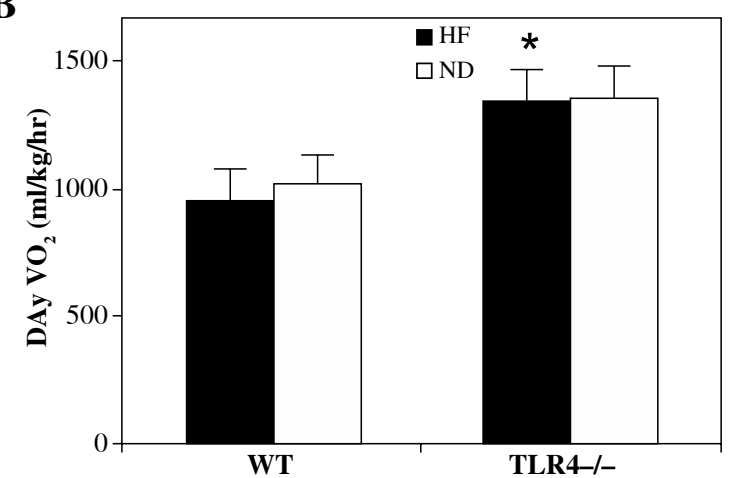

D
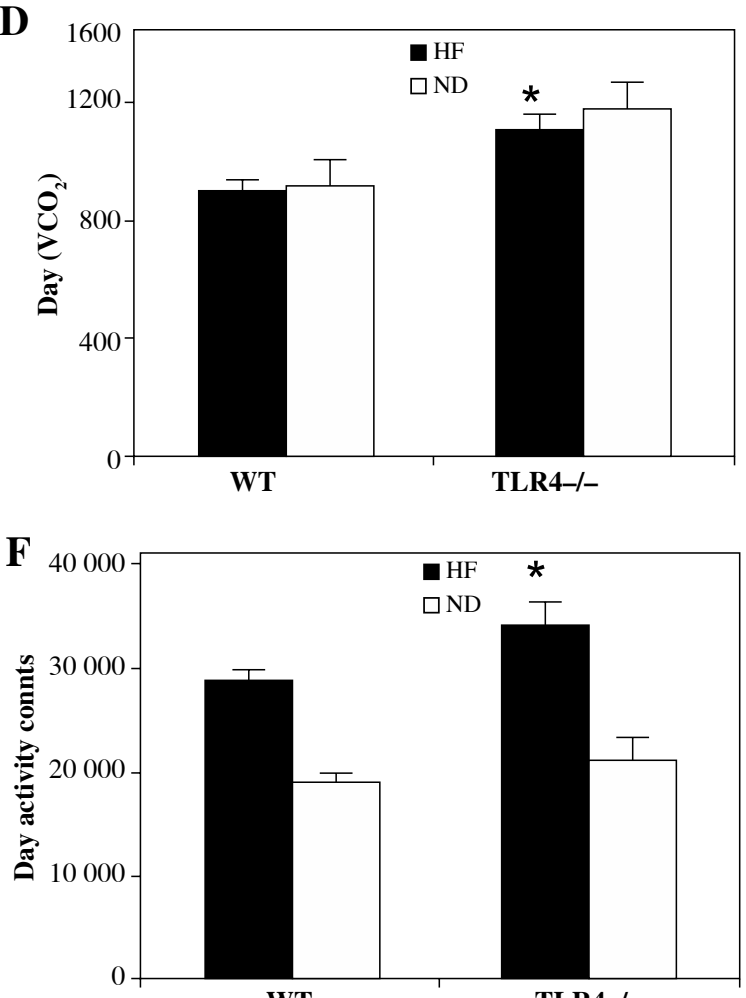

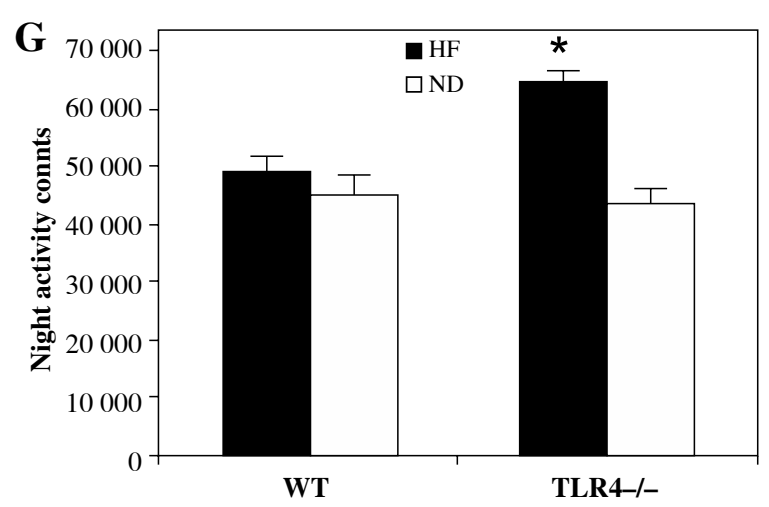

Fig. 2. In TLR4-/- HF mice, the $\mathrm{O}_{2}$ consumption, $\mathrm{CO}_{2}$ production and activity were higher than in the WT HF group. In the four groups, food intake (A), oxygen consumption during day $(\mathbf{B})$ and night $(\mathbf{C})$, the amount of emitted carbon dioxide during day $(\mathbf{D})$ and night $(\mathbf{E})$ and activities during day $(\mathbf{F})$ and night $(\mathbf{G})$ were measured. ${ }^{*} p<0.05 ;{ }^{* *} p<0.01$ 

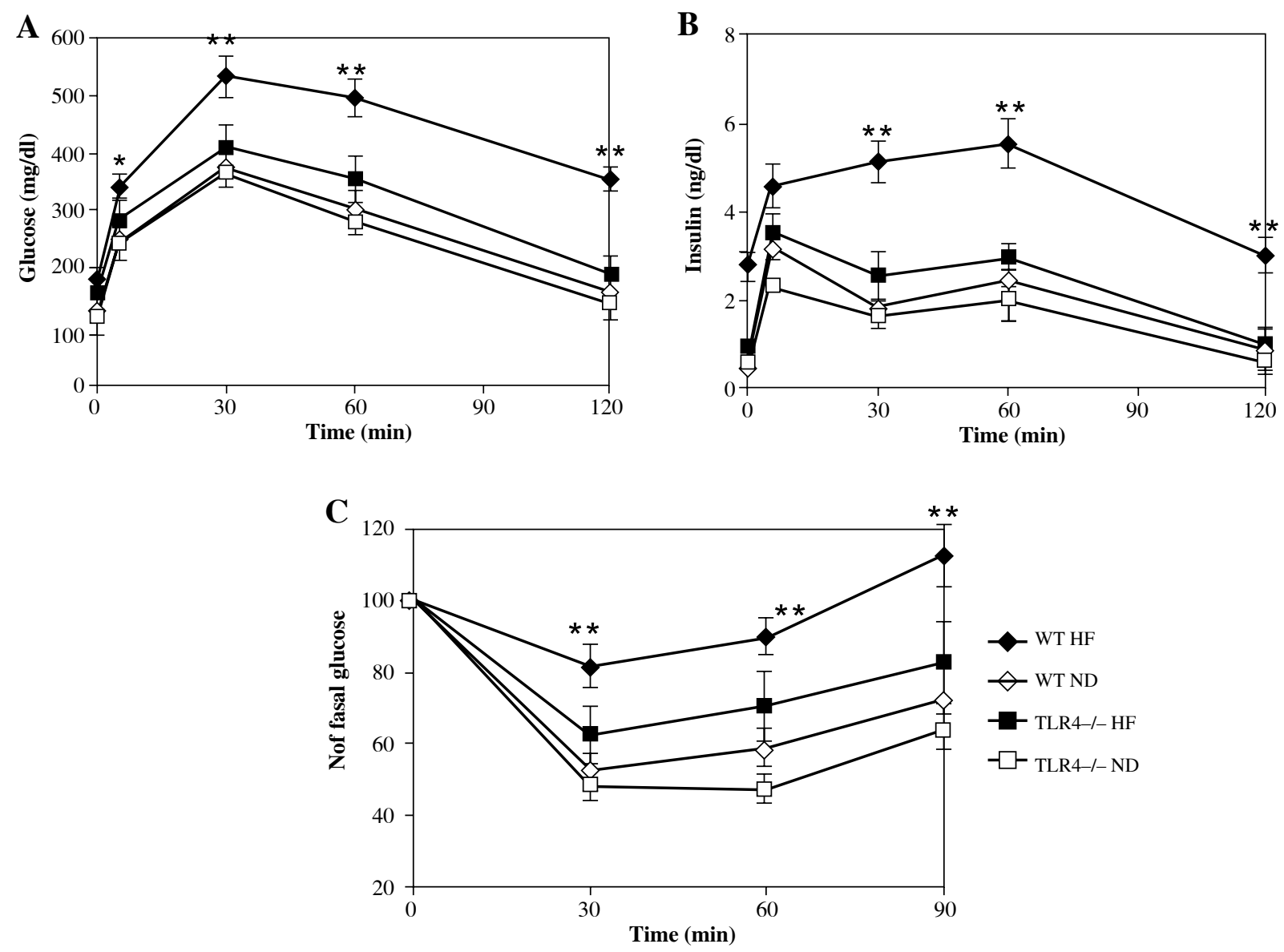

Fig. 3. The impaired insulin secretion was significantly improved in TLR4-/- HF mice, compared with the WT HF group, by the glucose tolerance test, insulin tolerance test and insulin release test. The glucose tolerance test (A), insulin release test $(\mathbf{B})$ and insulin tolerance test $(\mathbf{C})$ were done. ${ }^{*} p<0.05 ;{ }^{* *} p<0.01$

In the primary islet of TLR4-I- HF mice, by detecting glucose-stimulated insulin secretion, insulin secretion and the expression of inflammatory cytokines were changed, compared to those in the WT HF group

As shown in Fig. 5A, when given low glucose sugar of $2.8 \mathrm{mmol} / \mathrm{l}$, between the TLR4-/- HF group and WT $\mathrm{HF}$, there were no significant differences in insulin levels $(p>0.05)$, but after the high sugar $22 \mathrm{mmol} / \mathrm{l} \mathrm{stim-}$ ulation, the insulin level of the TLR4-/- HF group was significantly higher than that of the WT HF group, with a significant difference $(p<0.01)$. These results suggest that 24 weeks after continuous stimulation with the highfat diet, the functions of pancreatic $\beta$ cell in the WT HF group were significantly impaired, while the islet function of the TLR4-/- HF group was protected, and the islet glucose-stimulated insulin secretion (GSIS) function was associated with TLR4. And as shown in Fig. 5B, C, and
$\mathrm{D}$, after 24 weeks, in the WT HF group, at the mRNA level, islets IL-6, TNF- $\alpha$, and MCP-1 increased significantly higher than in the WT ND group $(p<0.05)$. But in the TLR4-/- HF group, after 24 weeks, islets IL-6, TNF- $\alpha$, and MCP-1 were significantly lower than those of the WT HF group at the mRNA level. These suggest that the highfat diet could promote islet inflammatory cytokine expression, whereas in TLR4 knockout, after the same high fat stimulation, the islet showed a low expression of inflammatory cytokines.

\section{In TLR4-/- HF mice, the islet macrophage infiltration was investigated, compared to that in the WT HF group}

As shown in Fig. 6, results from pancreatic islet immunostaining showed that pancreatic islet macrophage invasion was present in TLR4 WT mice fed with HF for 24 weeks, but not in TLR4-/- mice. 

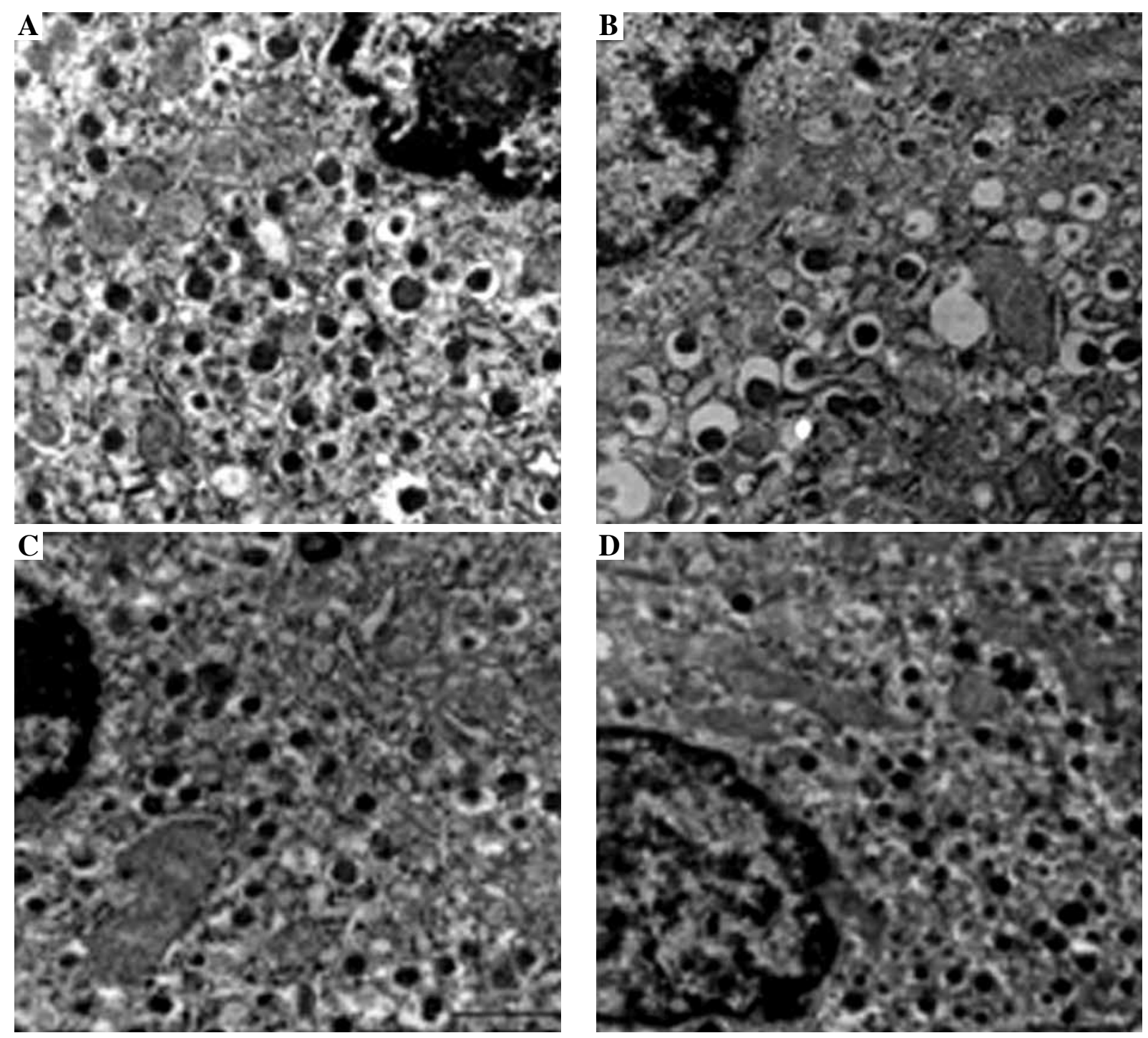

Fig. 4. In TLR4-I- HF mice, islet $\beta$ cell ultrastructure was not damaged in the face of the same high-fat continuous stimulation, compared to that in the WT HF group. The islet $\beta$ cell ultrastructure from the WT HF group (A), TLR4-/- HF group (B), WT ND group (C) and TLR4-/- ND group (D) were observed using electron microscopy

\section{Discussion}

Obesity is associated with chronic low-grade inflammation [15-17] that is characterized by increased circulating concentrations of proinflammatory cytokines [18, 19].

Insulin resistance is a primary defect leading to and a characteristic feature of diabetes [20]. The state of insulin resistance leads to increased insulin secretion by pancreatic $\beta$-cells and compensatory hyperinsulinemia. In patients destined to develop diabetes, $\beta$-cell compensation efficiency declines and relative insulin insufficiency develops leading to impaired glucose tolerance and eventually frank diabetes. Epidemiologic evidence demonstrates that patients with metabolic syndrome have a high likelihood of developing diabetes and cardiovascular disease. Thus, while it is well established that treatment of insulin resistance has beneficial effects in patients with diabetes, it is becoming increasingly clear that enhanced insulin sensitivity is also therapeutically important in nondiabetic individuals with insulin resistance.

Activation of the pro-inflammatory pathway has been described in a variety of insulin resistant states [21-24]. Chronic inflammation inhibits insulin sensitivity through the activation of signaling pathways that directly interfere with the normal function of key components of the insulin signaling pathway $[23,24]$. There are 11 members that have been identified to date in mammals. TLR1, TLR2, TLR4, TLR5, TLR6, TLR10, and TLR11 are expressed on 

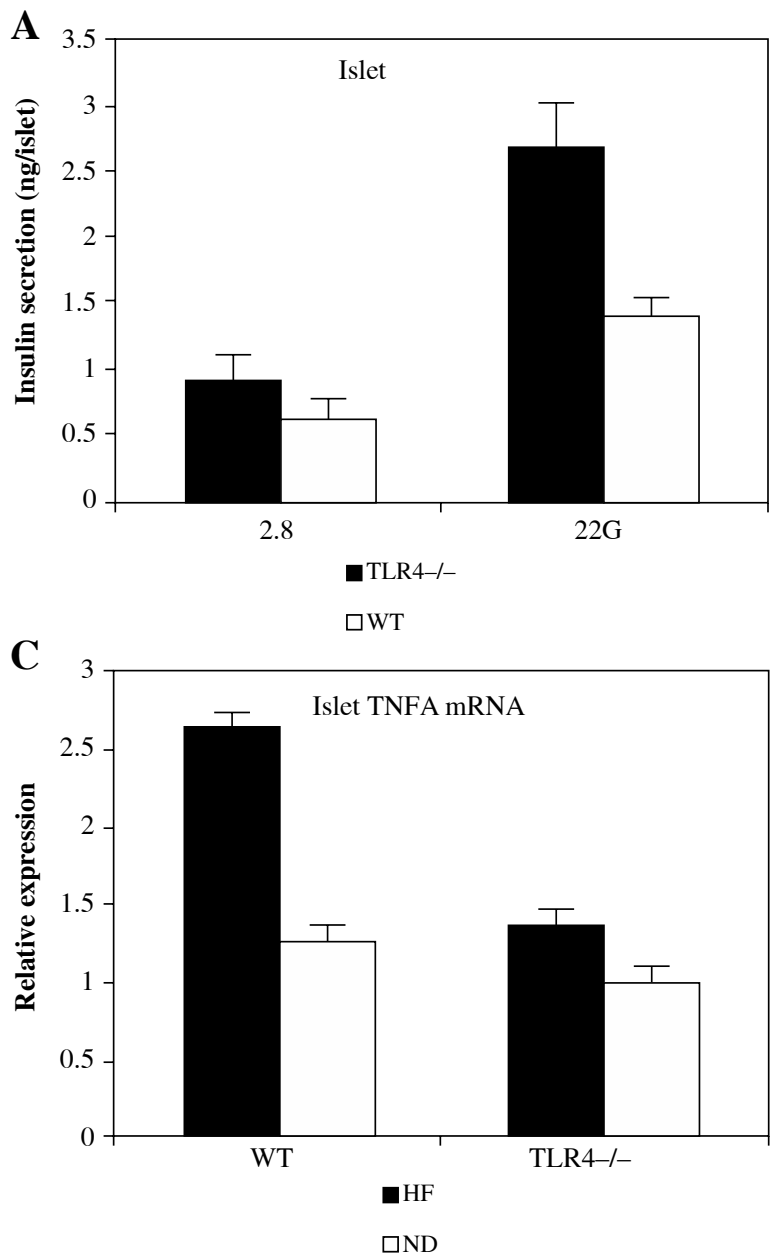

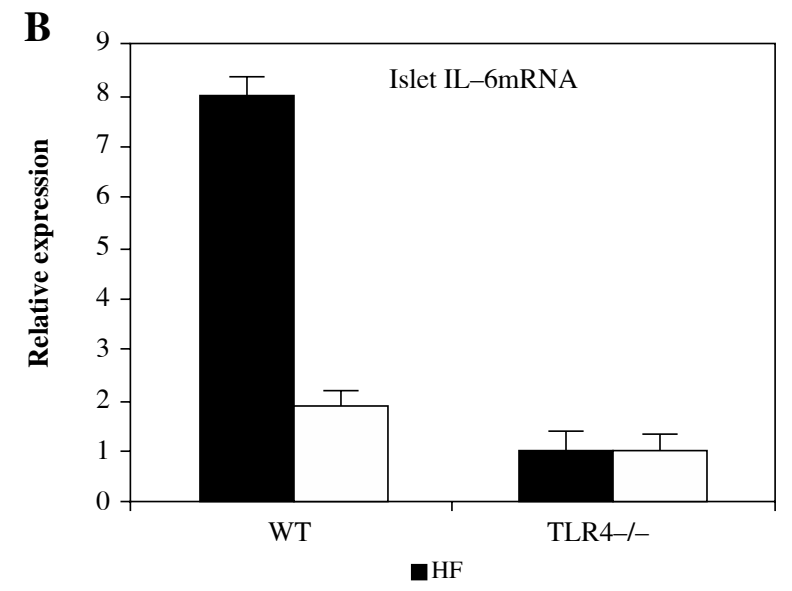

D

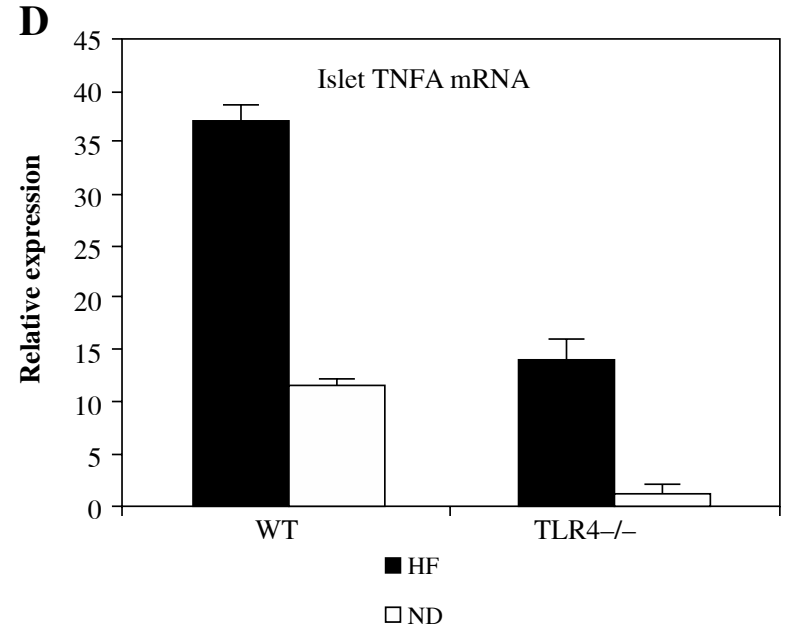

Fig. 5. In the primary islet, in TLR4-/- HF mice, by detecting glucose-stimulated insulin secretion, insulin secretion was better, and the expression of inflammatory cytokines was significantly lower, compared to those in the WT HF group. 24 weeks after high-fat diet, the separated mouse primary islets were given with $2.8 \mathrm{mmol} / \mathrm{l}$, glucose sugar or $22 \mathrm{mmol} / \mathrm{l}$ glucose, and insulin levels were measured by ELISA (A); islets IL-6 (B), TNF- $\alpha$ (C), and MCP-1 (D) were measured at the mRNA level

the cell surface, whereas TLR3, TLR7, TLR8, and TLR9 are expressed in intracellular compartments such as the endosome and the endoplasmic reticulum [25]. TLR2 and TLR4 may be directly involved in HF diet-induced inflammation and may also regulate basal and insulin-stimulated glucose uptake in adipocytes [26]. But the detailed mechanism for pancreatic $\beta$ cell dysfunction in diet-induced obesity is still unclear.

Therefore, we hypothesize that obesity may activate TLR4 and its signaling pathway, followed by recruitment of macrophage into pancreatic islet, which leads to up-regulation of downstream inflammatory factors, and finally results in pancreatic $\beta$ cell dysfunction.

TLR4 is a cell surface receptor that generates innate immune responses to pathogens by inducing signaling cascades of kinase and transcription factor activation. Previous studies have shown that FFA can act as the endoge- nous ligand for TLR4 to activate TLR4 in insulin sensitive tissues and cells including the liver, muscle, adipose, and macrophage. Activation of TLR4 signaling pathway leads to increased NF- $\kappa$ B activity, and finally results in insulin resistance [27]. There is also evidence showing that a high level of FFA can activate TLR4 signaling pathway and cause inflammatory reaction in vascular endothelial cells, which reduces the insulin sensitivity in vascular endothelial cells, and finally results in insulin resistance [28-30].

The data presented herein establish the relationship between obesity and TLR4. Our data showed that the glucose tolerance test, insulin tolerance test and insulin release test suggest that the impaired insulin secretion was significantly improved in TLR4-/- HF mice, compared with the WT HF group. While in TLR4-/- mice, there were no $\beta$ cell ultrastructure of the above changes, the deposition of lipid droplets obvious, and swelling of mitochondria, similar to 

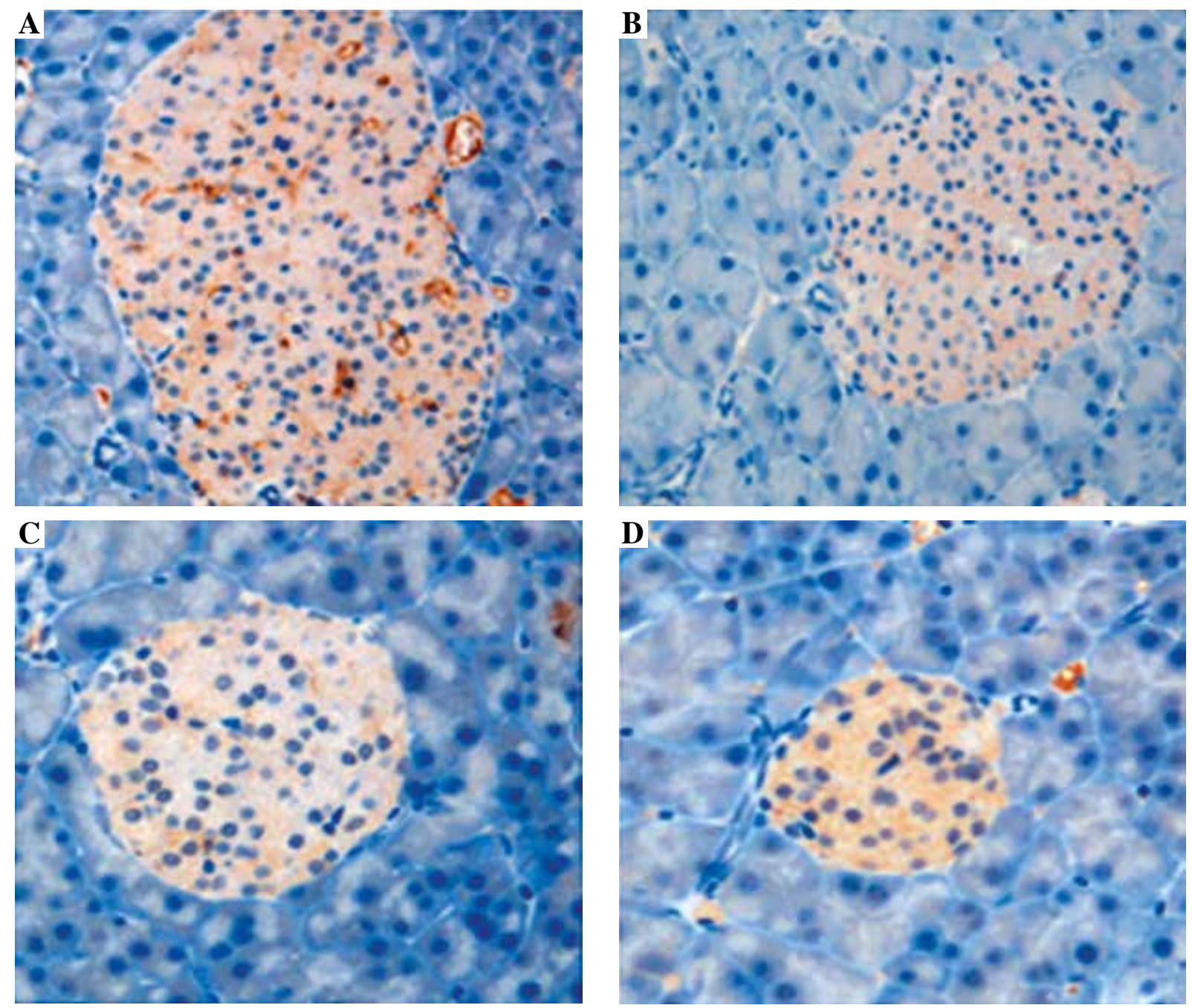

Fig. 6. In TLR4-/- HF mice, there was no significant macrophage infiltration, compared to that in the WT HF group. The macrophage infiltration in the islet from the WT HF group (A), TLR4-/- HF group (B), WT ND group (C) and TLR4-/- ND group (D) were observed using F480 immunohistochemical staining

$\beta$ cells of the normal diet group, suggesting that in TLR4-/HF mice, facing the same high-fat diet continuous stimulation, the islet $\beta$ cell ultrastructure was not damaged. Moreover, in the TLR4-/- HF group, IL-6, TNF- $\alpha$, and MCP-1 were significantly lower than in the WT HF group at the mRNA level. These data suggested that in TLR4 knockout, facing the same high fat stimulation, there was a low expression of inflammatory cytokines. Whereas the high-fat diets resulted in an increased MCP-1 expression in normal mice. Furthermore, the expression of both IL- 6 and TNF- $\alpha$ was markedly increased by the high-fat diets. These results indicate not only that some inflammatory responses to high-fat diets may be mediated independently of Tlr-4, at least at the mRNA level, but also that some responses to high fat intake occur. In addition, our data also showed that after continuous stimulation with the high-fat diet, the pan- creatic $\beta$ cell function of the wild-type WT HF group was significantly impaired, while the islet function of TLR4 knockout of the TLR4-/- HF group was protected, suggesting that the islet glucose-stimulated insulin secretion (GSIS) function was associated with TLR4.

In summary, our data showed that a loss-of-function point mutation in TLR4 could prevent diet-induced obesity, indicated that TLR4 was a key modulator in the crosstalk between inflammatory and metabolic pathways. We, therefore, suggest that a selective interference with TLR4 presents an attractive opportunity for the treatment of human obesity, insulin resistance, and diabetes.

This project was supported by the National Natural Science Foundation of China (31200887). 


\section{References}

1. Kim F, Tysseling KA, Rice J, et al. (2005): Free fatty acid impairment of nitric oxide production in endothelial cells is mediated by IKKbeta. Arterioscler Thromb Vasc Biol 25: 989-994.

2. Roden M, Price TB, Perseghin G, et al. (1996): Mechanism of free fatty acid-induced insulin resistance in humans. J Clin Invest 97: 2859-2865.

3. Shoelson SE, Lee J, Yuan M (2003): Inflammation and the IKK beta/I kappa B/NF-kappa B axis in obesity- and diet-induced insulin resistance. Int J Obes Relat Metab Disord 27: S49-S52.

4. Hotamisligil GS (2003): Inflammatory pathways and insulin action. Int J Obes Relat Metab Disord 27: S53-S55.

5. Cai D, Yuan M, Frantz DF, et al. (2005): Local and systemic insulin resistance resulting from hepatic activation of IKK-beta and NF-kappaB. Nat Med 11: 183-190.

6. Shoelson SE, Lee J, Goldfine AB (2006): Inflammation and insulin resistance. J Clin Invest 116: 1793-1801.

7. Francis K, Matilda P, Ian L, et al. (2007): Toll-Like receptor-4 mediates vascular inflammation and insulin resistance in diet-induced obesity. Circ Res 100: 1589-1596.

8. Kitchens RL, Ulevitch RJ, Munford RS (1992): Lipopolysaccharide (LPS) partial structures inhibit responses to LPS in a human macrophage cell line without inhibiting LPS uptake by a CD14-mediated pathway. J Exp Med 176: 485-494.

9. Munford RS, Hall CL (1986): Detoxification of bacterial lipopolysaccharides (endotoxins) by a human neutrophil enzyme. Science 234: 203-205.

10. Lee JY, Sohn KH, Rhee SH, Hwang D (2001): Saturated fatty acids, but not unsaturated fatty acids, induce the expression of cyclooxygenase-2 mediated through Toll-like receptor 4. J Biol Chem 276: 16683-16689.

11. Lee JY, Plakidas A, Lee WH, Heikkinen A, Chanmugam P, Bray G, Hwang DH (2003): Differential modulation of Tolllike receptors by fatty acids: preferential inhibition by $n-3$ polyunsaturated fatty acids. J Lipid Res 44: 479-486.

12. Hirabara SM, Silveira LR, Alberici LC, et al. (2006): Acute effect of fatty acids on metabolism and mitochondrial coupling in skeletal muscle. Biochim Biophys Acta 1757: 57-66.

13. Metze K, Andrade LA (1991): Atypical stromal giant cells of cervix uteri - evidence of Schwann cell origin. Pathol Res Pract 187: 1031-1035.

14. Xu H, Barnes GT, Yang Q, et al. (2003): Chronic inflammation in fat plays a crucial role in the development of obesity-related insulin resistance. J Clin Invest 112: 1821-1830.

15. Greenberg AS, Obin MS (2006): Obesity and the role of adipose tissue in inflammation and metabolism. Am J Clin Nutr 83: 461S-465S.

16. Neels JG, Olefsky JM (2006): Inflamed fat: what starts the fire? J Clin Invest 116: 33-35.

17. Wellen KE, Hotamisligil GS (2005): Inflammation, stress, and diabetes. J Clin Invest 115: 1111-1119.

18. Mantzoros CS, Moschos S, Avramopoulos I, et al. (1997): Leptin concentrations in relation to body mass index and the tumor necrosis factor-alpha system in humans. J Clin Endocrinol Metab 82: 3408-3413.

19. Roytblat L, Rachinsky M, Fisher A, et al. (2000): Raised interleukin-6 levels in obese patients. Obes Res 8: 673-675.

20. De Luca C, Olefsky JM (2008): Inflammation and insulin resistance. FEBS Letters 582: 97-105.

21. Olefsky JM, Glass CK (2010): Macrophages, inflammation, and insulin resistance. Ann Rev Physiol 72: 219-246.
22. Shah PK (2007): Innate immune pathway links obesity to insulin resistance. Circulation Research 100: 1531-1533.

23. Hotamisligil GS, Erbay E (2008): Nutrient sensing and inflammation in metabolic diseases. Nature Reviews Immunology 8: 923-934.

24. Schenk S, Saberi M, Olefsky JM (2008): Insulin sensitivity: modulation by nutrients and inflammation. J Clin Investigation 118: 2992-3002.

25. Kumar H, Kawai T, Akira S (2009): Toll-like receptors and innate immunity. Biochem Biophys Res Commun 388: 621625.

26. Davisa JE, Braucherb DR, Walker-Danielsb J, Spurlockb ME (2011): Absence of TIR2 protects against high-fat diet-induced inflammation and results in greater insulin-stimulated glucose transport in cultured adipocytes. J Nutr Biochem 22: 136-114.

27. Qi DF, Cai K, Wang QM, et al. (2010): Fatty acids induce amylin expression and secretion by pancreatic beta cells. Am J Physiol Endocrinol Metab 298: E99-E107.

28. Maeve AM, Orla MF, Ruth MC, et al. (2013): Mechanisms of obesity-induced inflammation and insulin resistance: insights into the emerging role of nutritional strategies. Front Endocrinol (Lausanne) 4: 52.

29. Orr JS, Puglisi MJ, Ellacott KL, et al. (2012): Toll-like receptor 4 deficiency promotes the alternative activation of adipose tissuemacrophages. Diabetes 61: 2718-2727.

30. Kim KA, Gu W, Lee IA, Joh EH, Kim DH (2012): High fat diet-induced gut microbiota exacerbates inflammation and obesity in mice via the TLR4 signaling pathway. PLoS One 7: e47713. 